\title{
Effect of connecting wires on the decoherence due to electron-electron interaction in a metallic ring
}

\author{
Christophe Texier ${ }^{1,2}$ \\ ${ }^{1}$ Laboratoire de Physique Théorique et Modèles Statistiques, \\ UMR 8626 du CNRS, Université Paris-Sud, 91405 Orsay, France. \\ ${ }^{2}$ Laboratoire de Physique des Solides, UMR 8502 du CNRS, Université Paris-Sud, 91405 Orsay, France.
}

(Dated: July 19, 2007)

\begin{abstract}
We consider the weak localization in a ring connected to reservoirs through leads of finite length and submitted to a magnetic field. The effect of decoherence due to electron-electron interaction on the harmonics of AAS oscillations is studied, and more specifically the effect of the leads. Two results are obtained for short and long leads regimes. The scale at which the crossover occurs is discussed. The long leads regime is shown to be more realistic experimentally.
\end{abstract}

PACS numbers: 73.23.-b ; 73.20.Fz ; 72.15.Rn

The classical Drude conductivity of metals is corrected at low temperature by small quantum corrections. One of them, called the weak localization (WL) correction, is a contribution to the average conductance due to interferences of reversed electronic trajectories. WL is a manifestation of quantum coherence, which provides a powerful tool to study phase coherence in weakly disordered metals. A possible way to measure $\mathrm{WL}$ is to analyze its magnetic field dependence : in particular the conductivity of a ring presents $\phi_{0} / 2$-periodic oscillations, as a function of the magnetic flux piercing the ring, called the Al'tshuler-Aronov-Spivak (AAS) oscillations $\frac{1,2}{1}\left(\phi_{0}=h / e\right.$ is the quantum flux). Measurement of AAS harmonics provides a central quantity of mesoscopic physics : the phase coherence length, the typical length over which electronic phase coherence is ensured.

Two articles ${ }^{3.4}$ have recently discussed how AAS harmonics of the WL correction to conductance $\left\langle\Delta g_{n}\right\rangle$ (or Aharonov-Bohm (AB) harmonics) of a metallic ring are affected by the decoherence due to electron-electron interaction. Ref $\frac{3}{3}$ emphasized the role to the wires connecting the ring to reservoirs, contrary to Ref $\stackrel{4}{=}$. This is the aim of the present paper to clarify this question.

We recall the basic framework necessary to study the question. We consider a metallic ring of perimeter $L$, connected at two reservoirs through leads of lengths $l_{a}$ (we consider a symmetric situation where the two arms of the ring have equal lengths $L / 2$ ). We define the geometrical parameter ${ }^{3} \gamma=\left(8 l_{a} / L+1\right)^{-1}$. AAS harmonics involve an integral of the cooperon $P_{c}^{(n)}(x, x)$ inside the network where wires are appropriately weighted ${ }^{4,5}$ :

$$
\left\langle\Delta g_{n}\right\rangle \simeq-8 \gamma^{2} \int_{\text {ring }} \frac{\mathrm{d} x}{L^{2}} P_{c}^{(n)}(x, x) .
$$

$P_{c}^{(n)}(x, x)$ encodes the contribution of closed interfering reversed electronic trajectories issuing from $x$ with winding $n$. In the present article we will be only interested in the limit of strong decoherence (high temperature $)^{6}$, therefore, since the harmonics of the cooperon are expected to vanish exponentially inside the leads on a scale given by the phase coherence length, contribu- tions $\int_{\text {leads }} \mathrm{d} x P_{c}^{(n)}$ of the connecting wires have been neglected in (11). Decoherence due to electron-electron interaction is taken into account within the framework of Ref. $\underline{\underline{Z}}$ : its efficiency is characterized by the Nyquist length $L_{N}=\left(\nu_{0} D^{2} / T\right)^{1 / 3}$ where $\nu_{0}$ is the density of states, $D$ the diffusion constant and $T$ the temperature. The cooperon can be written as a path integral

$$
\begin{array}{r}
P_{c}^{(n)}(x, x)=\int_{0}^{\infty} \mathrm{d} t \int_{x(0)=x}^{x(t)=x} \mathcal{D} x\left(t^{\prime}\right) \delta_{n, \mathcal{N}\left[x\left(t^{\prime}\right)\right]} \\
\times \mathrm{e}^{-\int_{0}^{t} \mathrm{~d} t^{\prime}\left[\frac{1}{4} \dot{x}^{2}+\frac{2}{L_{N}^{3}} W\left(x\left(t^{\prime}\right), x\left(t-t^{\prime}\right)\right]\right.},
\end{array}
$$

where $\mathcal{N}[x]$ is winding number of a diffusive trajectory around the ring. In principle path integral runs over trajectories $x\left(t^{\prime}\right)$ that explore the whole network, however in the limit of strong decoherence $\left(L_{N} \ll L\right)$ we will make the approximation that they remain inside the ring. Decoherence due to electron-electron interaction is modeled as phase fluctuations for a given electron due to coupling to the fluctuating electromagnetic field created by other electrons 7.8 .9 . Decoherence is accounted in (2) through the functional $\exp -\frac{2}{L_{N}^{3}} \int_{0}^{t} \mathrm{~d} t^{\prime} W$, where $W$ is related to the correlation function of electric potential (given by the fluctuation-dissipation theorem ${ }^{89} 9$ ). Therefore, decoherence depends on the network, and moreover on the nature of trajectories. In (11) the leads seem absent at first sight, however their effect is hidden in the cooperon, since the leads affect the nature of fluctuations of the electric potential, and therefore the decoherence, through $W$. It is the purpose of the present paper to discuss if it is an important effect or not. We will study successively the long and short lead limits. Finally we will obtain the scale of the crossover between the two regimes and their experimental relevance.

Long leads : $\gamma \simeq$ 0.- The difficulty in evaluating (12) lies in the time nonlocality of the action. In the case $\gamma=0$, translation invariance inside the ring is recovered, $W\left(x, x^{\prime}\right)=W\left(x-x^{\prime}, 0\right)$, what allows to get rid of time nonlocality thanks to $\underline{\underline{4}} W\left(x\left(t^{\prime}\right)-x\left(t-t^{\prime}\right), 0\right) \rightarrow$ $W\left(x\left(t^{\prime}\right), 0\right)$, and compute precisely the path integral. 
The cooperon is equivalent to the Green function describing tunneling of a particle with zero energy through periodic series of quadratic barriers, $V(x)=\frac{4}{L_{N}^{3}} W(x, 0) \propto$ $x\left(1-\frac{x}{L}\right)$ for $x \in[0, L]$, starting from $x=0$ and arriving at $x=n L$. For $L_{N} \ll L$, we have found in $\operatorname{Ref}^{4}$ :

$$
P_{c}^{(n)}(x, x) \sim L_{N} \mathrm{e}^{-n S_{\text {inst }}} \forall x \in \text { ring. }
$$

$S_{\text {inst }}=\frac{\pi}{8}\left(\frac{L}{L_{N}}\right)^{3 / 2}$ is the action of the tunneling trajectory through one barrier. The prefactor $L_{N}$ comes from the linear behaviour of the potential $W\left(x, x^{\prime}\right) \simeq \frac{1}{2}\left|x-x^{\prime}\right|$ at short distance, corresponding to $V(x) \propto|x|$ at the initial and final point for the tunneling trajectory $\underline{10}$. Finally the AAS harmonics of WL correction to the conductance read :

$$
\left\langle\Delta g_{n}\right\rangle \sim-\gamma^{2} \frac{L_{N}}{L} \mathrm{e}^{-n \frac{\pi}{8}\left(\frac{L}{L_{N}}\right)^{3 / 2}} .
$$

Harmonic of AB oscillations are related to AAS harmonics by $\stackrel{11}{\Perp}:\left\langle\delta g_{n}^{2}\right\rangle \sim-\gamma^{2}\left(\frac{L_{T}}{L}\right)^{2}\left\langle\Delta g_{n}\right\rangle$, where $L_{T}=\sqrt{D / T}$ is the thermal length, which leads to the temperature dependence $\left\langle\delta g_{n}^{2}\right\rangle \propto\left(\frac{L_{T}}{L}\right)^{8 / 3} \mathrm{e}^{-n S_{\text {inst }}}$. Two possible interpretations can be given to the result $\left\langle\Delta g_{n}\right\rangle \propto\left\langle\delta g_{n}^{2}\right\rangle \propto$ $\mathrm{e}^{-n S_{\text {inst }}}$. The scaling of harmonics with perimeter $L$ suggests to define a phase coherence length as $L_{\varphi} \rightarrow L_{N} \propto$ $T^{-1 / 3}$. On the other hand, assuming the $\mathrm{AAS}^{1}$ form $\left\langle\Delta g_{n}\right\rangle \propto \mathrm{e}^{-n L / L_{\varphi}}$, that is to consider the scaling with $n$, leads to define a $L$-dependent phase coherence length $L_{\varphi} \rightarrow \frac{8}{\pi} \frac{L_{N}^{3 / 2}}{L^{1 / 2}} \propto T^{-1 / 2}$. Physical interpretation of this new length scale have been given in Refs $\underline{3} \underline{3}$.

Short leads : $\gamma \neq \mathbf{0}$.- In this case $W$ is not anymore translation invariant inside the ring. To circumvent the problem of time nonlocality of the action, we can use the following procedure : for $t^{\prime} \in[0, t / 2]$ we introduce $x_{1}\left(t^{\prime}\right)=x\left(t^{\prime}\right)$ and $x_{2}\left(t^{\prime}\right)=x\left(t-t^{\prime}\right)$. Thanks to the property $W\left(x, x^{\prime}\right)=W\left(x^{\prime}, x\right)$ we get :

$$
\begin{aligned}
P_{c}^{(n)}(x, x)=2 \int_{0}^{\infty} \mathrm{d} t \int_{\operatorname{ring}} \mathrm{d} x^{\prime} \int_{x_{1}(0)=x}^{x_{1}(t)=x^{\prime}} \mathcal{D} x_{1}\left(t^{\prime}\right) \int_{x_{2}(0)=x}^{x_{2}(t)=x^{\prime}} \mathcal{D} x_{2}\left(t^{\prime}\right) \\
\times \delta_{n, \mathcal{N}\left[x_{1}, x_{2}\right]} \mathrm{e}^{-S\left[x_{1}, x_{2}\right]}
\end{aligned}
$$

where $\mathcal{N}\left[x_{1}, x_{2}\right]$ is the winding number of the two trajectories put end to end. The action $S\left[x_{1}, x_{2}\right]=$ $\int_{0}^{t} \mathrm{~d} t^{\prime}\left[\frac{1}{4}\left(\dot{x}_{1}^{2}+\dot{x}_{2}^{2}\right)+\frac{4}{L_{N}^{3}} W\left(x_{1}\left(t^{\prime}\right), x_{2}\left(t^{\prime}\right)\right)\right]$ describes a $2-$ dimensional problem, local in time. In the high temperature regime of interest here $\left(L_{N} \ll L\right)$ we can use semiclassical methods. Path integral (5) is dominated by classical trajectories of winding $n$. Such trajectories are solutions of the classical equation of motion $\ddot{\vec{r}}=\frac{8}{L_{N}^{3}} \vec{\nabla} W$ on the torus $[0, L] \times[0, L]$, where $\vec{r} \equiv\left(x_{1}, x_{2}\right)$, for initial and final conditions $\vec{r}(0)=(x, x)$ and $\vec{r}(t)=\left(x^{\prime}, x^{\prime}\right)$, with zero energy [hamiltonian is $\left.\mathcal{H}=-\frac{1}{4} \dot{\vec{r}}^{2}+\frac{4}{L_{N}^{3}} W(\vec{r})\right]$. To simplify the problem we can set $L=L_{N}=1$, and denote by $\widetilde{S}$ the new dimensionless action, since at zero energy the $L / L_{N}$ dependence of the action is easily recovered : $S_{\mathcal{H}=0}=\left(\frac{L}{L_{N}}\right)^{3 / 2} \widetilde{S}_{\mathcal{H}=0}$. At zero energy, the initial condition on the line $x_{1}=x_{2}$, such that $W=0$, fully determines the classical trajectory. Therefore we expect the structure $P_{c}^{(n)}(x, x) \propto \mathrm{e}^{-n\left(\frac{L}{L_{N}}\right)^{3 / 2} \widetilde{S}^{\mathrm{cl}}(x / L)}$, where $\widetilde{S}^{\mathrm{cl}}(x)$ is the action of the classical trajectory issuing from $\vec{r}(0)=(x, x)$ with winding $n=1\left(\right.$ for $\left.L=L_{N}=1\right)$.
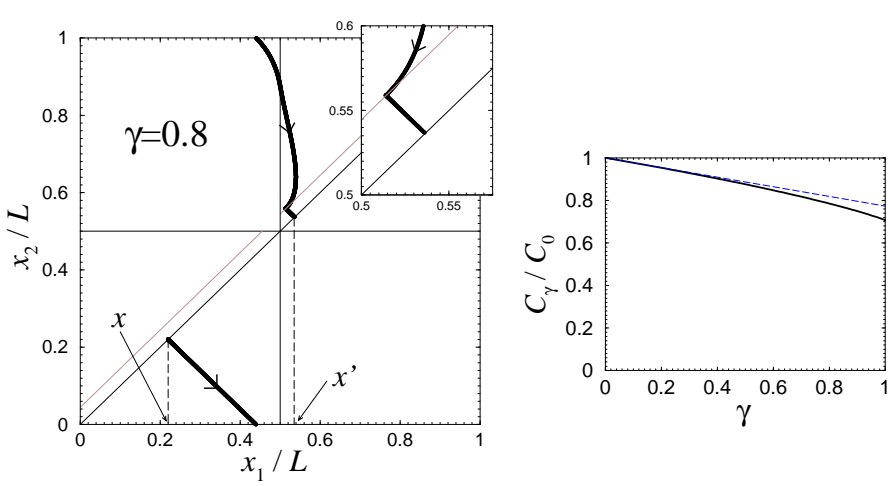

FIG. 1: Left : Example of a classical trajectory of winding $n=1$. The last small segment (inset) corresponds to tunneling : coordinates aquire a complex part not represented.

Thanks to the symmetries of potential, we can easily find two particular solutions of the equation of motion. (i) The solution on the line $x_{1}+x_{2}=L / 2($ or $3 L / 2)$ is the one with minimal action $\widetilde{S}^{\mathrm{cl}}(1 / 4)=\frac{C_{\gamma}}{C_{0}} \frac{\pi}{8}$. The dimensionless parameter is ${ }^{3}: C_{\gamma}=\left(\frac{\pi}{2(\gamma+1)}\right)^{3 / 2}[\pi+2 \arcsin \gamma+$ $2 \gamma \sqrt{1-\gamma^{2}}$. The ratio $C_{\gamma} / C_{0}$ varies monotonously between 1 and $C_{1} / C_{0}=1 / \sqrt{2}$ (Fig. (1) . (ii) The solution on the line $x_{1}+x_{2}=0$ (or $L$ ) is the one with maximal action $\widetilde{S}^{\mathrm{cl}}(0)=\frac{\pi}{8}$. Apart for initial conditions $x=0, L / 4$, $L / 2$ or $3 L / 4$, the trajectory aquires some finite speed $v_{\|}=\frac{\dot{x}_{1}+\dot{x}_{2}}{\sqrt{2}}$ in the direction parallel to the line $x_{1}=x_{2}$. Therefore it can only wind around the ring by going to complex time $t^{\prime}= \pm \mathrm{i} \tau$. Action also aquires some complex part, $\widetilde{S}^{\mathrm{cl}}(x)=S_{\text {real }}(x)+\mathrm{i} S_{\text {imag }}(x)$, and can be studied numerically (Fig. 2). (There are two complex trajectories for the two possible signs $t^{\prime}= \pm \mathrm{i} \tau$, with conjugated actions).

Finally, assuming the structure

$$
P_{c}^{(n)}(x, x) \sim L_{N} \mathrm{e}^{-n\left(\frac{L}{L_{N}}\right)^{3 / 2} \widetilde{S}^{\mathrm{cl}}(x / L)},
$$

where we have kept the same preexponential factor $L_{N}$ as for the case $\gamma=0$ since potential presents the same short distance behaviour $W \simeq \frac{1}{2}\left|x-x^{\prime}\right|$. The cooperon is maximal at the middle of the arms of the ring $(x=L / 4$ and $3 L / 4$ ). Assuming Gaussian fluctuations and using the steepest descent approximation, we obtain :

$$
\left\langle\Delta g_{n}\right\rangle \sim-\frac{\gamma^{2}}{\sqrt{n}}\left(\frac{L_{N}}{L}\right)^{7 / 4} \mathrm{e}^{-n \frac{C_{\gamma}}{C_{0}} \frac{\pi}{8}\left(\frac{L}{L_{N}}\right)^{3 / 2}} .
$$

Now we write $S_{\text {inst }}=\frac{C_{\gamma}}{C_{0}} \frac{\pi}{8}\left(\frac{L}{L_{N}}\right)^{3 / 2}$. Integration over $x$ has brought an additional factor $\left(L_{N} / L\right)^{3 / 4}$. Corresponding $\mathrm{AB}$ harmonics are $\left\langle\delta g_{n}^{2}\right\rangle \propto\left(\frac{L_{T}}{L}\right)^{19 / 6} \mathrm{e}^{-n S_{\text {inst }}}$. 
Despite we follow the same ideas as LM, $\frac{3}{\underline{3}}$ our preexponential factors for $\mathrm{AB} / \mathrm{AAS}$ amplitudes disagree $\underline{10}$ with LM's results $\left\langle\Delta g_{n}\right\rangle_{\mathrm{LM}} \sim L_{N}^{9 / 4} \mathrm{e}^{-n S_{\text {inst }}}$ and $\left\langle\delta g_{n}^{2}\right\rangle_{\mathrm{LM}} \sim$ $L_{T}^{7 / 2} \mathrm{e}^{-n S_{\text {inst }}}$, by a factor $\left(L_{N} / L\right)^{1 / 2} \propto T^{-1 / 6}$.

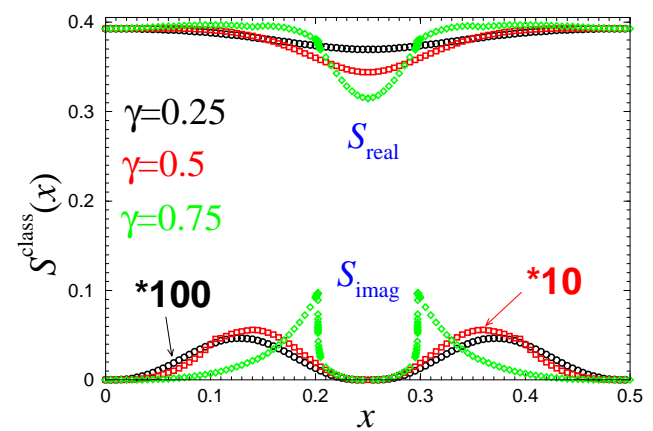

FIG. 2: Real (top curves) and imaginary (bottom curves) parts of the action of classical trajectory as a function of initial condition $x$, for $\gamma=0.25$ (black circles), $\gamma=0.5$ (red squares) and $\gamma=0.75$ (green diamonds). Imaginary parts for $\gamma=$ 0.25 and $\gamma=0.5$ have been rescaled by factors 100 and 10, respectively.

Crossover.- The steepest descent method, used to derive (7), requires $\left(1-\frac{C_{\gamma}}{C_{0}}\right) \frac{\pi}{8}\left(\frac{L}{L_{N}}\right)^{3 / 2} \gg 1$. In the opposite limit, $P_{c}^{(n)}(x, x)$ can be considered almost constant inside the ring and eq. (4) holds. Therefore, since $\frac{C_{\gamma}}{C_{0}} \simeq 1-0.23 \gamma$ (where $\frac{3}{2}-\frac{4}{\pi} \simeq 0.23$ ), the crossover between these two situations occurs for $\gamma \sim \gamma_{c}$ where

$$
\gamma_{c} \simeq 11\left(\frac{L_{N}}{L}\right)^{3 / 2}
$$

Discussion.- To summarize, we have obtained two expressions (47) and (7) for the AAS harmonics, corresponding to long $\left(\gamma \lesssim \gamma_{c}\right)$ and short leads $\left(\gamma \gtrsim \gamma_{c}\right)$ regime. Their derivations assumed : (i) $L_{N} / L \ll 1$ (validity of semiclassical approximation), (ii) $L_{N} / l_{a} \ll 1$, where $l_{a}$ is the length of the leads, otherwise some other $L_{N}$-dependent preexponential factor is expected ${ }^{4,13}$ since winding trajectories feel absorbing reservoirs. From experimental point of view, observability of the effect requires (iii) $L_{N} / L$ large enough in order to obtain some signal. Applicability of long leads result (4) is $(i v) l_{a} / L$ large $\left(\gamma \lesssim \gamma_{c}\right)$. On the other hand, applicability of the short leads result (7) requires : (v) $l_{a} / L$ small enough $\frac{l_{a}}{L} \lesssim \frac{1}{8}\left(\frac{1}{\gamma_{c}}-1\right)$, what is more problematic. Let us rewrite contraints $(i i) \&(v)$ as $\frac{l_{a}}{L_{N}} \gtrsim 1$ and $\frac{l_{a}}{L_{N}} \lesssim \frac{1}{8} \frac{L}{L_{N}}\left[0.09\left(\frac{L}{L_{N}}\right)^{3 / 2}-1\right]$. This is only possible for $L_{N} / L \lesssim 1 / 8$, which seems pretty irrealistic from experi- mental point of view since it corresponds to a suppression of AAS harmonics of $\mathrm{e}^{-\frac{\pi}{8}\left(\frac{L}{L_{N}}\right)^{3 / 2}} \approx 10^{-4}$.

Let us consider a real situation and use parameters corresponding to experiments of Ref $\underline{\underline{14}}$. The highest temperature at which AAS oscillations were still observable $\frac{15}{}$ was $T=700 \mathrm{mK}$ (the Nyquist length was $L_{N} \simeq 0.59 \mu \mathrm{m}$ at $1 \mathrm{~K}$ ) at which $L_{N} / L \simeq 0.17$, which is above the threshold of applicability of the short leads result (7). At this temperature $\gamma_{c} \simeq 0.7$, which leaves a large room to the long leads regime (4), and for which $(i i)$ is not possible to fulfill since $(v)$ gives $l_{a} \lesssim 0.2 \mu \mathrm{m}$ far below $L_{N}=0.66 \mu \mathrm{m}$. However we note that, up to now, the experiment $\frac{15}{15}$ is not able to give information on the $T$-dependent prefactor.

We conclude that the long leads result (4) might be used for devices easily realized, whereas short leads result (77) seems to apply to rather irrealistic situations.

Acknowlegments.- I thank Gilles Montambaux and Denis Ullmo for interesting discussions and comments.

Appendix : potential $\boldsymbol{W}\left(\boldsymbol{x}, \boldsymbol{x}^{\prime}\right)$.- The potential is defined ${ }^{4}$ as $W\left(x, x^{\prime}\right)=\frac{1}{2}\left[P_{d}(x, x)+P_{d}\left(x^{\prime}, x^{\prime}\right)\right]-P_{d}\left(x, x^{\prime}\right)$, where the diffuson $P_{d}$ is solution of $-\Delta P_{d}\left(x, x^{\prime}\right)=$ $\delta\left(x-x^{\prime}\right)$ and vanishes at the reservoirs. By definition it is a symmetric function of its two arguments. It has fully been constructed for a connected ring in Ref $\underline{\underline{4}}$. We choose coordinate $x \in[0, L]$ such that $x=0$ and $x=L / 2$ correspond to the points of attachment of the leads.

- If $x, x^{\prime} \in$ the same arm $W\left(x, x^{\prime}\right)=\frac{1}{2}\left|x-x^{\prime}\right|[1-(\gamma+$ 1) $\left.\frac{\left|x-x^{\prime}\right|}{L}\right]$. In the plane $\left(x, x^{\prime}\right)$, equipotentials are straight lines.

- If $x, x^{\prime} \notin$ the same arm. For $0 \leqslant x \leqslant L / 2 \leqslant x^{\prime} \leqslant L$ : $W\left(x, x^{\prime}\right)=\frac{1}{2}\left(x^{\prime}-x\right)\left[1-(\gamma+1) \frac{x^{\prime}-x}{L}\right]-\frac{2 \gamma}{L}\left(x-\frac{L}{2}\right)\left(x^{\prime}-\frac{L}{2}\right)$. Equipotentials are ellipses (circles for $\gamma=1$ ). Note that on the line $x+x^{\prime}=L$ we find that $W(x, L-x)=x\left(1-\frac{2 x}{L}\right)$ is independent on $\gamma$.

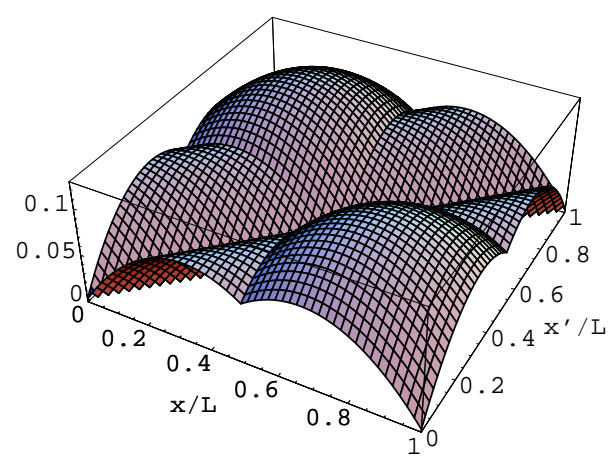

FIG. 3: Potential $W\left(x, x^{\prime}\right)$ as a function of $x / L$ and $x^{\prime} / L$, for $\gamma=0.5$.
1 B. L. Al'tshuler, A. G. Aronov, and B. Z. Spivak, The Aaronov-Bohm Effect in disordered conductors, JETP Lett. 33(2), 94 (1981).
${ }^{2}$ D. Yu. Sharvin and Yu. V. Sharvin, Magnetic-flux quantization in a cylindrical film of a normal metal, JETP Lett. 34(5), 272 (1981). 
3 T. Ludwig and A. D. Mirlin, Interaction-induced dephasing of Aharonov-Bohm oscillations, Phys. Rev. B 69, 193306 (2004).

4 C. Texier and G. Montambaux, Dephasing due to electronelectron interaction in a diffusive ring, Phys. Rev. B 72, 115327 (2005) ; ibid 74, 209902(E) (2006).

${ }^{5}$ C. Texier and G. Montambaux, Weak localization in multiterminal networks of diffusive wires, Phys. Rev. Lett. 92, 186801 (2004).

6 The high temperature regime (phase coherence length larger than perimeter) has been studied in Ref.4.

7 B. L. Altshuler, A. G. Aronov, and D. E. Khmelnitsky, Effects of electron-electron collisions with small energy transfers on quantum localisation, J. Phys. C: Solid St. Phys. 15, 7367 (1982).

8 B. L. Altshuler and A. G. Aronov, Electron-electron interaction in disordered conductors, in Electron-electron interactions in disordered systems, edited by A. L. Efros and M. Pollak, page 1, North-Holland, 1985.

9 E. Akkermans and G. Montambaux, Mesoscopic physics of electrons and photons, Cambridge University Press, 2007.

10 In Ref ${ }^{3}$, LM have unproperly used semiclassical methods to estimate preexponential factor. In particular they did not account for the fact that initial/final point in path integral are turning points where WKB approximation fails.

11 As noticed in appendix E of Ref. $\stackrel{4}{*}$, the relation between $\mathrm{AB}$ and $\mathrm{AAS}$ harmonics is not universal but holds when the role of the wire weights introduced in Ref. ${ }^{5}$ can be neglected. For the symmetric connected ring considered here, we distinguish two limits. (1) $L_{\varphi} \gg L$ : quantum correction is dominated by integrals over the leads and the relation $\left\langle\delta g_{n}^{2}\right\rangle \simeq-\frac{16 \pi}{3} \gamma^{2}\left(\frac{L_{T}}{L}\right)^{2}\left\langle\Delta g_{n}\right\rangle \simeq-\frac{\pi}{3}\left(\frac{L_{T}}{2 l_{a}}\right)^{2}\left\langle\Delta g_{n}\right\rangle$ is exactly similar as for a long wire ${ }^{12}$. (2) $L_{\varphi} \ll L$ : quantum correction is dominated by integrals inside the ring, whose arms have a relative weight $1 / 4$ compare to the leads : $\left\langle\delta g_{n}^{2}\right\rangle \simeq-\frac{4 \pi}{3} \gamma^{2}\left(\frac{L_{T}}{L}\right)^{2}\left\langle\Delta g_{n}\right\rangle$. These relations are valid for $L_{\varphi} \gg L_{T}$, which is always the case if electron-electron is the dominant mechanism ${ }^{8}$ described by the phase coherent length $L_{\varphi}$.

12 I. L. Aleiner and Ya. M. Blanter, Inelastic scattering time for conductance fluctuations, Phys. Rev. B 65, 115317 (2002).

13 C. Texier and G. Montambaux, Quantum oscillations in mesoscopic rings and anomalous diffusion, J. Phys. A: Math. Gen. 38, 3455 (2005).

14 M. Ferrier, L. Angers, A. C. H. Rowe, S. Guéron, H. Bouchiat, C. Texier, G. Montambaux, and D. Mailly, Direct measurement of the phase coherence length in a GaAs/GaAlAs square network, Phys. Rev. Lett. 93, 246804 (2004).

15 M. Ferrier, L. Angers, A. C. H. Rowe, S. Guéron, H. Bouchiat, C. Texier, G. Montambaux, and D. Mailly, Geometrical dependence of the decoherence due to electronic interactions in a GaAs/GaAlAs square network, (2007), in preparation. 